\title{
Decision Support for Off-the-Shelf Software Selection in Web Development Projects
}

\author{
Widura Schwittek and Stefan Eicker (PhD supervisor) \\ paluno - The Ruhr Institute for Software Technology \\ University of Duisburg-Essen \\ Universitätsstr. 9, 45141 Essen, Germany \\ \{widura.schwittek|stefan.eicker\}@paluno.uni-due.de
}

\begin{abstract}
Reusing off-the-shelf (OTS) components (including commercial and open source software) has become a key success factor in software development projects leading to reduced costs, faster time-to-market and better software quality. This especially holds true in the field of web engineering, where software engineers are faced with a steady proliferation of new technologies and standards. But there are upfront and ongoing efforts and risks attached to the adoption of OTS components which makes decision-making very difficult. Such difficulties are for example a large and intransparent market, incompatibilities between components and architectural mismatches. In this paper, a plan towards a novel platform concept is proposed that can improve the situation for software engineers coping with the adoption of OTS components during webbased systems development.

One key contribution is an empirically derived ontology to describe software artifacts on a feature level allowing a better description and identification of OTS components in the domain of web development. Another key contribution is a concept for a lean experience sharing mechanism. The goal of both contributions is to improve OTS component decision-making.

The concept will be implemented as a platform prepopulated with OTS components from the domain of Java web development. A cross-case study is planned to evaluate the concept.
\end{abstract}

Keywords: off-the-shelf components, open source software, web engineering, web-based systems development, decision support, experience management.

\section{Introduction}

Reusing off-the-shelf (OTS) components has become a key success factor in software development projects [1] leading to reduced costs, faster time-to-market and better software quality [2]. This especially holds true in the field of web engineering, where architects and developers are faced with the steady proliferation of new technologies and standards [3]. The term "OTS components" is considered as an umbrella term which includes commercial off-the-shelf (COTS) components and Open Source Software (OSS), such as in [4]. OTS components are ready-to-use software components 
which can be acquired commercially (COTS) or free of charge (OSS) and are easily reusable because of their cohesive nature. OTS components are being reused in software development projects and therefore address software engineers rather than endusers. In my work, I consider OTS component types such as frameworks, libraries, or UI component sets.

If software engineers decide to build upon OTS components, they are confronted with a large and vibrant OTS component marketplace, especially in the domain of web-based systems development. This provides many opportunities, but difficulties as well:

- Information about OTS components is cluttered around several sources (websites, brochures, etc.) and has often not the quality to serve as a foundation for decision-making.

- Many alternatives to the same problem exist with slight differences which need to be identified.

- OTS component decisions can impact other decisions leading to complex interdependencies.

In all, OTS component selection becomes a time-consuming and tedious activity.

In this paper, a plan towards a novel platform concept is proposed that can improve the situation for software engineers coping with the adoption of OTS components during web-based systems development and evolution. In the following, this group of software engineers will be called "integrators".

\section{Key Contributions}

In the following two sections, the two key contributions of this research is described in more detail, each contributing to a "decision support for off-the-shelf software selection in web development projects" and addressing the shortcomings of the related work presented in the previous section.

\subsection{A Feature Ontology for Enhancing OTS Component Descriptions}

The main goal of this part of the research is the development of an ontology to be able to semantically enrich the description of OTS components from the domain of webbased systems development on a feature level. If OTS components are described in this way, they can be searched more elaborately solving issues such as the use of different but synonym search terms or inferring aspects the integrator was not aware of in the first place.

The ontology focuses on features of OTS components. Features comprise external and internal features. External features directly satisfy customer's requirements such as the feature "Scrollable Data Table" of a UI component suite. This allows a mapping of requirements onto OTS component features. Overlapping or missing features can be easily identified. Internal features are of interest for the actual integration of 
the OTS component such as "Dependency Injection" or "MVC". Internal features are also of use for discovering architectural mismatches.

In order to derive the ontology an empirical study is planned, for which a large and representative set of OTS components from the web development domain will be analyzed. The analysis process is repeated until additional OTS components do not significantly contribute to the feature ontology anymore. All harvested feature descriptions will then have to be harmonized according to the different terms used. I will focus on the OTS component market for Java web development which is known to be quite cluttered and in which the author already has practical experience. The study will be conducted systematically so that it can be repeated for other programming languages and even for software development domains other than web development.

The feature ontology will be implemented within a proof-of-concept tool serving two use cases. First, it will support describing OTS components on a feature level in an accurate and unambiguous way. Second, it will support identifying OTS components in this tool providing an exploratory (faceted) search and a comparison mechanism. The second use case will be evaluated as part of a cross-case study, proving or disproving the effectiveness of such an ontology for identifying OTS components.

\subsection{Sharing OTS Component Selection Experience}

During the time-consuming and tedious activity of OTS component selection, wellthought decisions as well as bad decisions are made over and over again. Experience about this is highly valuable but is not often shared, although it is available. Asking people who already solved a similar problem or developed a web application in a similar setting are a popular and welcomed source of information to prepare a decision.

The main goal of this part of my research is the extension of the tool described in the previous section. While it already provides means to enrich OTS component descriptions with ontology-based features, the tool should also incorporate OTS component selection experience. For this, the OTS component description facility will be extended by a "used by" statistic and inferred recommendations. It is assumed that the experience of one's OTS component decision is of interest for someone else. The "used by" data is generated by other integrators using the platform for managing their OTS component selection. Recommendations are generated from underlying data mining mechanisms.

Additionally, further means to provide feedback is planned allowing to rate relations between OTS components. As an example, these ratings might indicate incompatibilities between different components or successful integrations.

Rules can be generated on basis of this data, which is either provided upfront by experts or is inferred from existing combinations and feedback by other integrators. This makes it possible to automatically detect valid or invalid combinations, similar to a configurator used for cars.

A final OTS component selection can be marked as "final", thus contributing to the overall knowledge of successful OTS component combinations. This final selection can be enhanced by decision rationales contributing to the architectural knowledge 
which is of relevance during evolution and maintenance of the software system as well. As a byproduct, it serves as a starting point for a software architecture documentation, to be handed over to stakeholders for discussion or to be used in bulletin boards, so that people know, what OTS component set the integrator is talking or asking about.

The evaluation of these "experience sharing" features of the tool is difficult, since a critical mass of users is required to reach an amount of data which is generalizable. In the case the critical mass is reached, an online questionnaire will be used to evaluate the concept. If the critical mass is not reached, it is planned to provide experience data through investigating large Open Source projects and extract OTS component combinations out of these. Then, a cross-case study will be used to evaluate the concept.

\section{Related Work}

Many repository and search engine approaches have been proposed ([5],[6],[7]) to support finding the right OTS components within the numerous OTS components available and matching the requirements. Also, much research can be observed in the fields of component specification and standardization ([8],[9]). Nonetheless, studies have shown that rather than really using these approaches during OTS component identification, the most usual way in practice is to ask colleagues relying on the experience of experts or use general internet search engines [2]. Our platform implementing the feature ontology as well as the experience exchange mechanisms aims at enhancing the repository approach making it more useful and thus more attractive for practitioners.

Furthermore, most of the research which can be found in this context is dedicated to component models such as CORBA, EJB, JavaBeans etc. which is not the theme of this work. As already mentioned, I refer to a broader meaning of OTS components, also including frameworks, programming libraries, UI component suites etc.

An increasing body of knowledge on Semantic Web Enabled Software Engineering can be observed, which also becomes visible through an ISWC collocated workshop series. But only little research deals with semantically enriching OTS component descriptions: "KOntoR" [10] provides an extendable ontology for software reuse, but keeps quite general in terms of a specific domain and thus lacks of a concrete application. The "OTS wiki" focuses on sharing OTS knowledge following the Wiki approach [11]. Our research is seeking for a feature ontology for describing OTS for the web engineering domain and for knowledge sharing mechanisms other than offered by Wikis.

A huge body of knowledge exists in the context of "feature models" originating from Software Product Line Engineering (SPLE). While there are differences between "feature models" and "ontologies" [12], this research is strongly related when it comes to using our feature ontology for OTS identification. Further investigation towards this direction is therefore planned. 


\section{Work Already Done, and a Tentative Plan}

The author already conducted research in the fields of architectural knowledge sharing [13] and ontologies for codifying bodies of knowledge [14]. Although the author has made no experience yet in the scientific field of web engineering, he already participated as software architect in industry projects, where he got the inspiration for this research.

This $\mathrm{PhD}$ work will be conducted cumulatively. While there are two big parts clearly defined (as described in section 3.1 and section 3.2) I see enough challenges for even smaller parts resulting in one paper each. Currently starting my third year as PhD student, I plan to finish my work in 2014.

\section{Literature}

1. Gartner: The Evolving Open-source Software Model (Predicts from December 2008)

2. Li, J., Conradi, R., Bunse, C., Torchiano, M., Slyngstad, O.P.N., Morisio, M.: Development with Off-the-Shelf Components: 10 Facts. IEEE Softw. 26(2), 80-87 (2009), doi:10.1109/MS.2009.33

3. Rossi, G.: Web engineering: Modelling and implementing web applications. Human computer interaction series, vol. 12. Springer, London (2008)

4. Ayala, C., Hauge, Ø., Conradi, R., Franch, X., Li, J.: Selection of third party software in Off-The-Shelf-based software development: An interview study with industrial practitioners. Journal of Systems and Software 84(4), 620-637 (2011), doi:10.1016/j.jss.2010.10.019

5. Clark, J., Clarke, C., de Panfilis, S., Granatella, G., Predonzani, P., Sillitti, A., Succi, G., Vernazza, T.: Selecting components in large COTS repositories. Journal of Systems and Software 73(2), 323-331 (2004), doi:10.1016/j.jss.2003.09.019

6. Wanyama, T., Far, B.: Repositories for Cots Selection. In: Canadian Conference on Electrical and Computer Engineering, Ottawa, ON, Canada, May 7-10, pp. 2416-2419. IEEE Service Center, Piscataway (2006), doi:10.1109/CCECE.2006.277301

7. Yanes, N., Sassi, S.B., Ghezala, H.H.B.: State of Art and Practice of COTS Components Search Engines. In: IEEE/ACS International Conference on Computer Systems and Applications, AICCSA 2010, Hammamet, Tunisia, May 16-19, pp. 1-8. IEEE, Piscataway (2010), doi:10.1109/AICCSA.2010.5587032

8. Cechich, A., Requile-Romanczuk, A., Aguirre, J., Luzuriaga, J.: Trends on COTS Component Identification. In: Proceedings of the Fifth International Conference on Commercialoff-the-Shelf (COTS)-Based Software Systems, ICCBSS 2006, Orlando, Florida, February 13-16, pp. 90-99. IEEE Computer Society, Los Alamitos (2006), doi:10.1109/ICCBSS.2006.31

9. Crnkovic, I., Sentilles, S., Vulgarakis, A., Chaudron, M.R.V.: A Classification Framework for Software Component Models. IEEE Trans. Software Eng. 37(5), 593-615 (2011), doi:10.1109/TSE.2010.83

10. Happel, H., Korthaus, A., Seedorf, S., Tomczyk, P.: KOntoR: An Ontology-enabled Approach to Software Reuse

11. Li, J., Ayala, C., Conradi, R.: Role-Based Wiki for Reuse of Off-the-Shelf Components

12. Czarnecki, K., Kim, C.H.P., Kalleberg, K.T.: Feature Models are Views on Ontologies 
13. Schwittek, W., Eicker, S.: Communicating Architectural Knowledge: Requirements for Software Architecture Knowledge Management Tools. In: Babar, M.A., Gorton, I. (eds.) ECSA 2010. LNCS, vol. 6285, pp. 457-463. Springer, Heidelberg (2010)

14. Schwittek, W., Schmidt, H., Eicker, S., Heisel, M.: Towards a Common Body of Knowledge for Engineering Secure Software and Services. In: Filipe, J., Liu, K. (eds.) KMIS 2011 - Proceedings of the International Conference on Knowledge Management and Information Sharing, Paris, France, October 26-29, pp. 369-374. SciTePress (2011) 